\title{
HECHTIA MAPIMIANA (BROMELIACEAE; HECHTIOIDEAE), UNA NUEVA ESPECIE DEL ESTADO DE DURANGO, MÉXICO
}

\author{
Ana Rosa López-Ferrari y Adolfo Espejo-Serna ${ }^{1}$ \\ Universidad Autónoma Metropolitana-Iztapalapa, División de \\ Ciencias Biológicas y de la Salud, Departamento de Biología, \\ Apdo. postal 55-535, 09340, México, D. F. \\ 1Autor para la correspondencia: aes@xanum.uam.mx
}

\section{RESUMEN}

Se describe e ilustra Hechtia mapimiana una nueva especie endémica del Cañón de Fernández, sierra de Mapimí, en el estado de Durango, México. El nuevo taxon se compara con $H$. zamudioi, con la cual presenta similitudes a nivel vegetativo y floral.

Palabras clave: Bromeliaceae, Durango, Hechtia, México.

\begin{abstract}
Hechtia mapimiana a new endemic species from Cañón de Fernández, sierra de Mapimí, in the state of Durango, Mexico, is described and illustrated. The new taxon is compared with $H$. zamudioi, species with similar vegetative and floral characteristics.
\end{abstract}

Key words: Bromeliaceae, Durango, Hechtia, Mexico.

Como parte del proyecto Flora Bromeliológica Mexicana, nos hemos dado a la tarea de recolectar material de los diferentes géneros de la familia en diversas localidades del país. En septiembre de 2005 encontramos, en el estado de Durango, material femenino de un taxon no descrito de Hechtia y recientemente recibimos material de intercambio procedente del herbario del Centro Interdisciplinario de Investigación para el Desarrollo Integral Regional, Unidad Durango, Instituto Politécnico Nacional (CIIDIR), en el cual se incluía el correspondiente ejemplar mas- 
culino, de manera que hemos podido completar adecuadamente la descripción de la especie que aquí proponemos como:

Hechtia mapimiana López-Ferrari et Espejo sp. nov. Figs. 1, 2A, 3A, 4A y 4C

Lithophytic rosulated herbs, to $2.3 \mathrm{~m}$ high; leaves glaucous, conic-cylindrical at the apex, sheaths pale brown on the adaxial surface, dark brown on the abaxial one; pistillate inflorescences twice branched; staminate inflorescences twice or thrice branched, with apparently fascicled racemes; staminate and pistillate flowers pale green coloured, filaments in the male flowers 3.2-3.5 mm long, anthers 4.2-4.5 $\mathrm{mm}$ long, green; capsules papyraceous, ovoid, ca. $1 \mathrm{~cm}$ long, $8-9 \mathrm{~mm}$ in diameter.

Hierbas rupícolas arrosetadas, cespitosas, en flor de hasta $2.3 \mathrm{~m}$ de alto, las rosetas actinomorfas, de ca. $35 \mathrm{~cm}$ de alto, de 40 a $70 \mathrm{~cm}$ de diámetro, formando colonias de pocos individuos; tallos inconspicuos. Hojas numerosas, carnosas, las vainas pardas claras en el haz, pardas oscuras en el envés, ampliamente ovadas a subcuadradas, de 6 a $15 \mathrm{~cm}$ de largo, de 5.5 a $10 \mathrm{~cm}$ de ancho, lustrosas, glabras en ambas superficies, las láminas de color glauco, larga y angostamente triangulares, cilíndrico-cónicas y ligeramente curvadas hacia arriba en la parte apical, de 30 a $45 \mathrm{~cm}$ de largo, de 2.5 a $4 \mathrm{~cm}$ de ancho en la base, densamente blanco-lepidotas en ambas superficies y con pelos marginales delgados y blancos, espinosas en los márgenes y con una corta pero conspicua espina apical de color pardo rojizo, las espinas marginales curvadas hacia la base y pardas claras a rojizas en el ápice, de 3 a $6 \mathrm{~mm}$ de largo, separadas entre sí 2 a $5 \mathrm{~cm}$. Inflorescencias terminales, erectas, compuestas, paniculadas; la estaminada de ca. $2 \mathrm{~m}$ de alto, dos a tres veces dividida, con numerosas ramas, éstas agrupándose en fascículos aparentes por la reducción del raquis, el pedúnculo cilíndrico, de ca. 1.5 de largo, de 2.5 a $3 \mathrm{~cm}$ de diámetro; brácteas del pedúnculo foliáceas, las vainas enteras, ovadotriangulares, papiráceas, quebradizas, de 4.5 a 5.5 de largo, de 2.6 a $3.2 \mathrm{~cm}$ de ancho, muy esparcidamente blanco-lepidotas a glabras, las láminas angostamente triangulares, de 8 a $10 \mathrm{~cm}$ de largo, de 5 a $7 \mathrm{~mm}$ de ancho, espinosas en el margen, pungentes en el ápice, densamente blanco-lepidotas en ambas superficies; ramas de primer orden oblongo-cilíndricas, de 5.4 a $6.8 \mathrm{~cm}$ de largo, con dos ramas basales de segundo orden, de 2.8 a $4.5 \mathrm{~cm}$ de largo; brácteas primarias pardas claras, papiráceas, ovado-triangulares, de 5 a $6 \mathrm{~cm}$ de largo, de 2.5 a $3 \mathrm{~cm}$ de ancho, conspicuamente nervadas, largamente acuminadas en el ápice; brácteas florales filiformes, blancas, de 3 a $5 \mathrm{~mm}$ de largo, más cortas que los pedicelos, enteras, 
López-Ferrari y Espejo-Serna: Hechtia mapimiana (Bromeliaceae), nueva especie de Durango, México
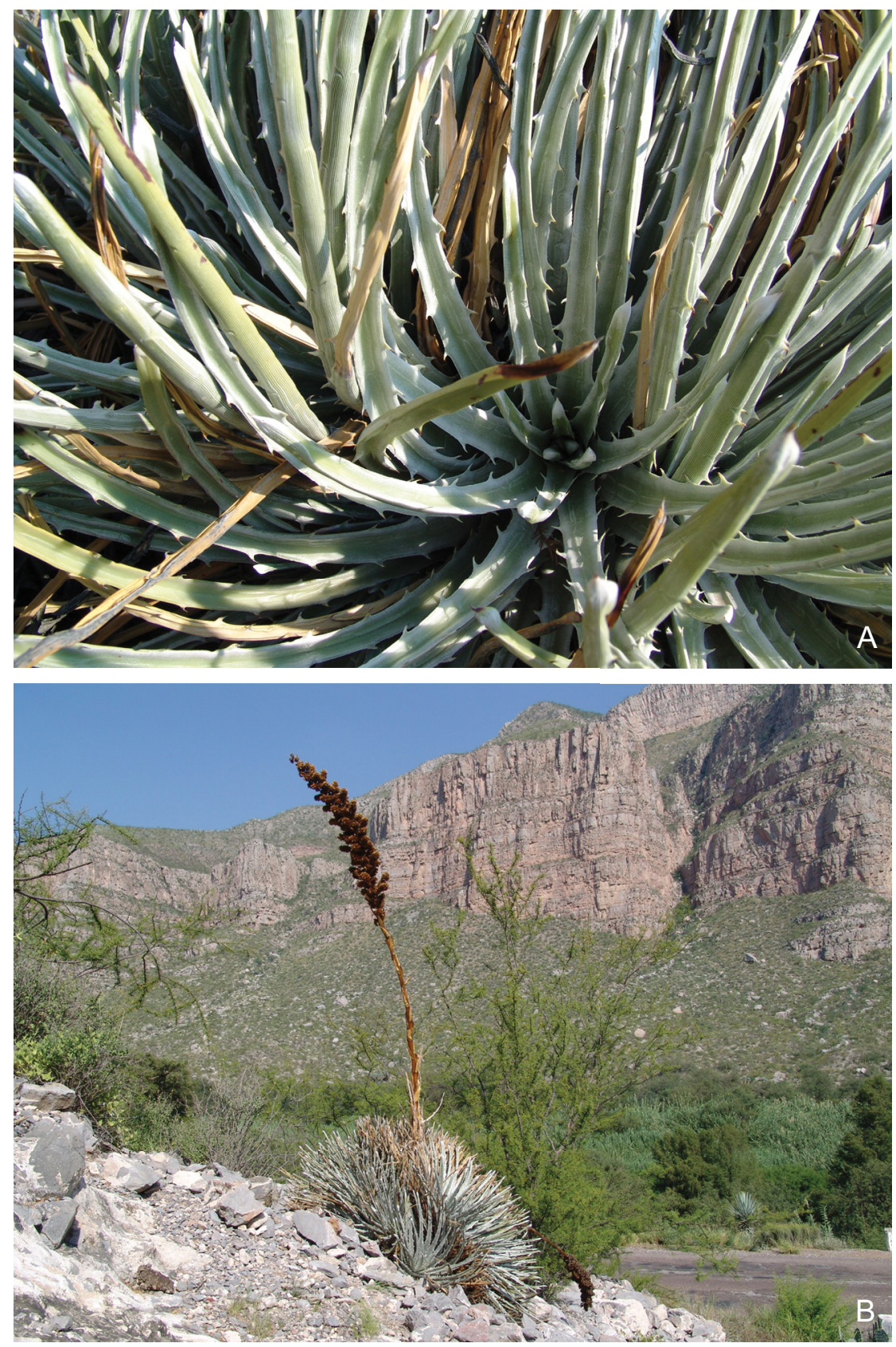

Fig. 1. Hechtia mapimiana López-Ferrari et Espejo. A. Roseta. B. colonia de rosetas femeninas (J. Ceja et al. 1691). Fotografías A. Espejo. 
glabras; flores polísticas, ascendentes, pedicelos filiformes, de 5.5 a $11 \mathrm{~mm}$ de largo; sépalos libres, membranáceos, triangulares, de 3.6 a $4 \mathrm{~mm}$ de largo, de 1.5 a 1.7 $\mathrm{mm}$ de ancho, blancos con tintes rosados a purpúreos, agudos en el ápice; pétalos libres, membranáceos, elípticos, de 6.5 a $7.5 \mathrm{~mm}$ de largo, de 4 a $4.2 \mathrm{~mm}$ de ancho, verdes claros, redondeados en el ápice; estambres subiguales, filamentos lineartriangulares, de 3.2 a $3.5 \mathrm{~mm}$ de largo, blancos, anteras angostamente oblongas, de 4.2 a $4.5 \mathrm{~mm}$ de largo, verdes; ovario vestigial; la inflorescencia pistilada de ca. $2.3 \mathrm{~m}$ de alto, dos veces dividida, con numerosas ramas, éstas agrupándose en fascículos aparentes por la reducción del raquis, el pedúnculo cilíndrico, de 1.7 a 1.8 $\mathrm{m}$ de largo, de 2.5 a $3 \mathrm{~cm}$ de diámetro; brácteas del pedúnculo foliáceas, las vainas enteras, ovado-triangulares, papiráceas, quebradizas, de 5 a $6 \mathrm{~cm}$ de largo, de 2.8 a $3 \mathrm{~cm}$ de ancho, muy esparcidamente blanco-lepidotas a glabras; las láminas angostamente triangulares, de 7 a $16.5 \mathrm{~cm}$ de largo, de 5 a $7 \mathrm{~mm}$ de ancho, espinosas en el margen, pungentes en el ápice, densamente blanco-lepidotas en ambas superficies; ramas de primer orden oblongo-cilíndricas, de 6 a $9 \mathrm{~cm}$ de largo, con dos flores solitarias en la base; brácteas primarias pardas claras, papiráceas, triangulares, de 4 a $7 \mathrm{~cm}$ de largo, de 1.5 a $1.7 \mathrm{~cm}$ de ancho, glabras, conspicuamente nervadas, largamente pungentes en el ápice; brácteas florales filiformes, blancas, de 3 a $5 \mathrm{~mm}$ de largo, más cortas que los pedicelos, enteras, glabras; flores polísticas, ascendentes, pedicelos filiformes, de 7 a $8 \mathrm{~mm}$ de largo; sépalos libres, membranáceos, triangulares, de $3.5 \mathrm{~mm}$ de largo, de $2.5 \mathrm{~mm}$ de ancho, blancos con tintes rosados a purpúreos, agudos en el ápice; pétalos libres, membranáceos, ovado-triangulares, de 5 a $5.2 \mathrm{~mm}$ de largo, de 2.5 a $2.7 \mathrm{~mm}$ de ancho, verdes claros, agudos en el ápice; estaminodios con los filamentos linear-triangulares, de ca. $2.5 \mathrm{~mm}$ de largo, blancos, anteras ausentes; ovario ovoide; cápsulas pardas claras, ovoides, triquetras, de ca. $1 \mathrm{~cm}$ de largo, de 8 a $9 \mathrm{~mm}$ de diámetro, con las paredes delgadas, papiráceas; semillas no vistas.

Tipo: México, Durango, municipio de Lerdo, Cañón de Fernández, presa Francisco Zarco, matorral xerófilo, 1450 m s.n.m., 12.V.2008, planta masculina, $F$. Mercado, D. Ramírez y N. López 259 (holotipo: CIIDIR, isotipos: IEB, UAMIZ).

Paratipos: México. Durango, municipio de Lerdo, parque estatal Cañón de Fernández, ca. $3 \mathrm{~km}$ después de Nuevo Graseros, rumbo a la presa Francisco Zarco, matorral xerófilo, 1745 m s.n.m., 7.IX.2005, planta femenina, J. Ceja Romero, A. Espejo, A. R. López-Ferrari, A. Mendoza R. e I. Ramírez M. 1691 (CIIDIR, IEB, UAMIZ); municipio de Lerdo, ca. 24 (air) miles SW of Torreón, in a narrow canyon, in vertical 
López-Ferrari y Espejo-Serna: Hechtia mapimiana (Bromeliaceae), nueva especie de Durango, México
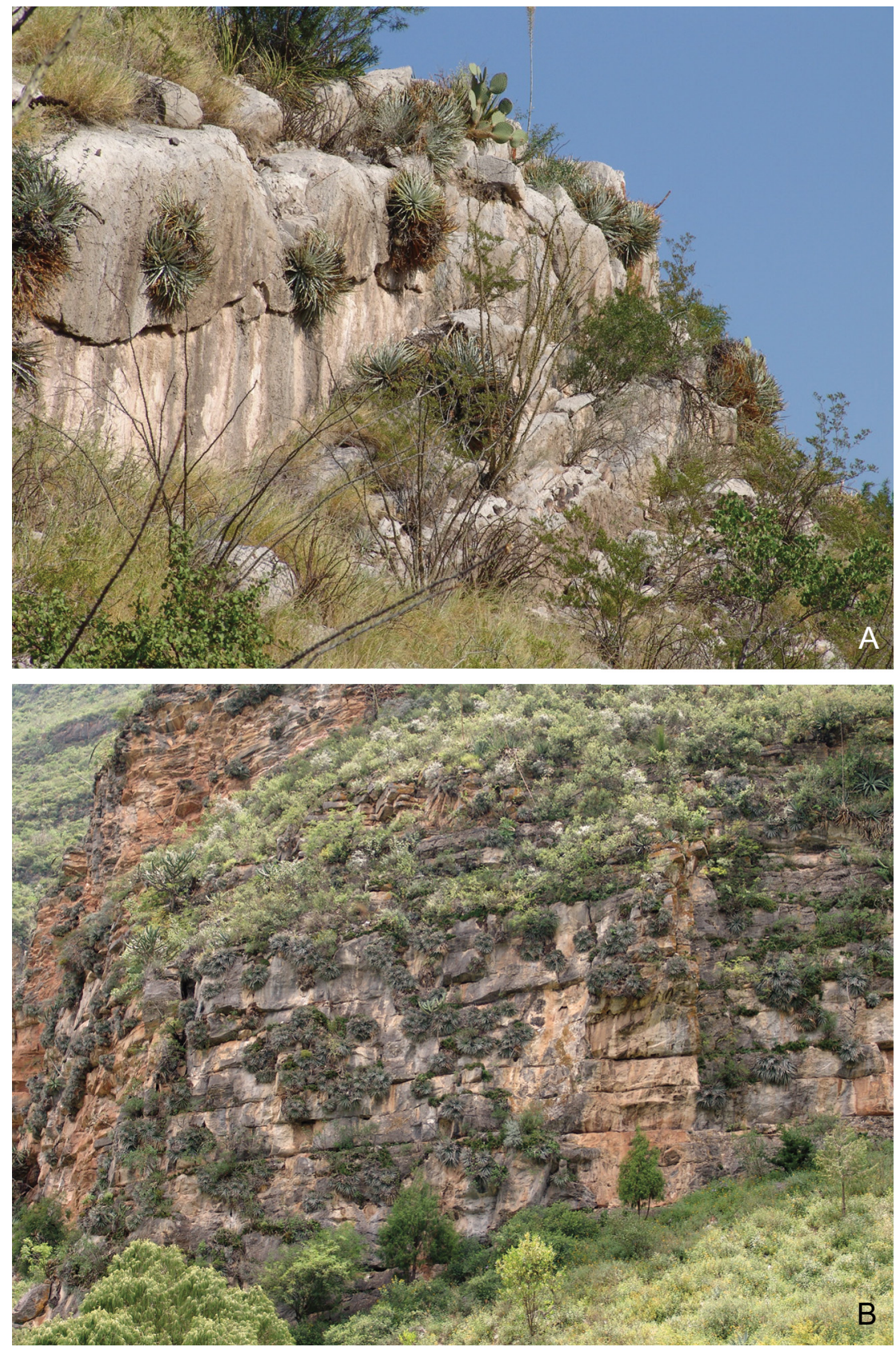

Fig. 2. H. mapimiana López-Ferrari et Espejo (A) y H. zamudioi Espejo, López-Ferrari et I. Ramírez (B) en su hábitat. Fotografías A. Espejo. 

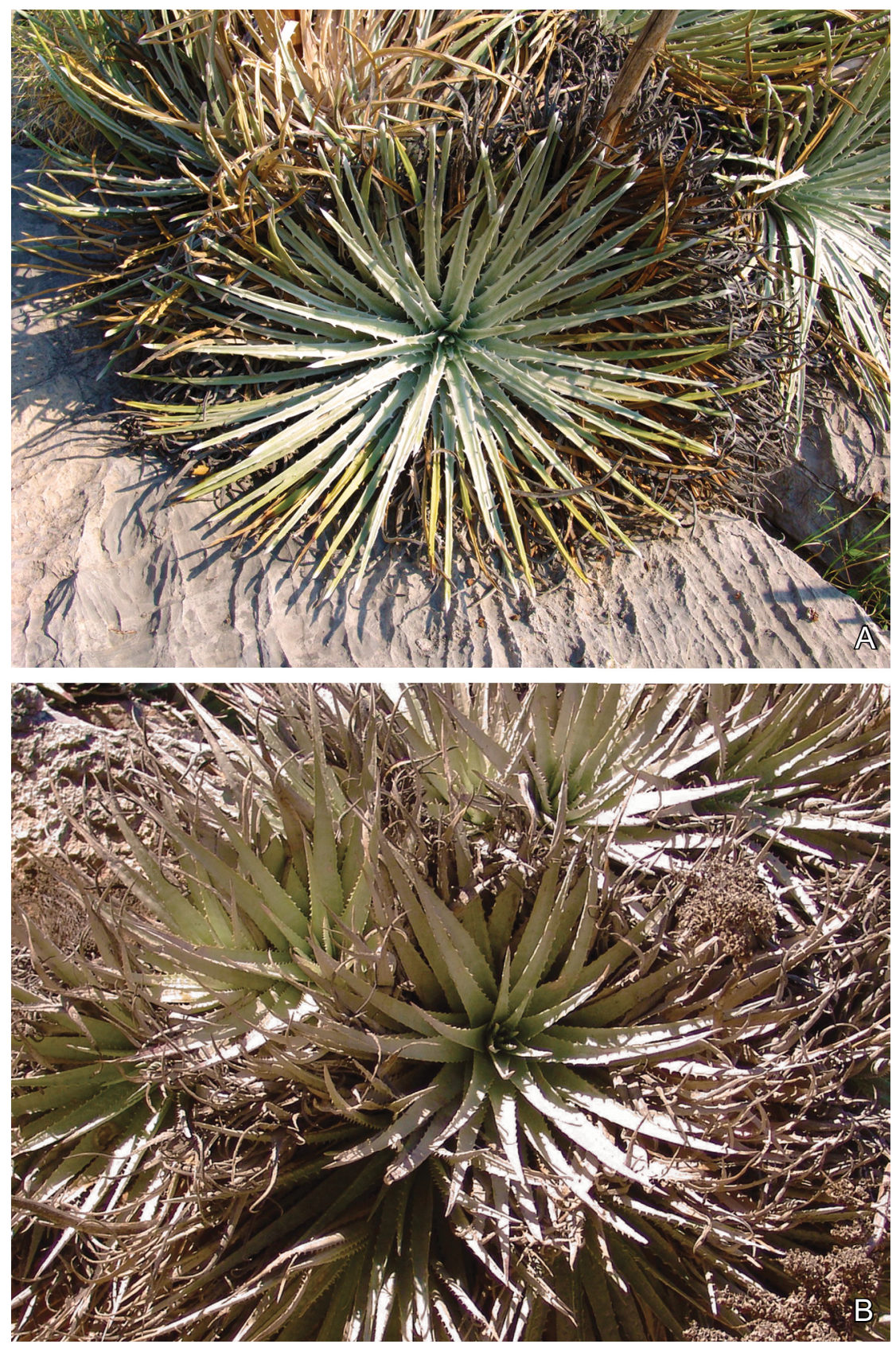

Fig. 3. Rosetas de: (A) Hechtia mapimiana López-Ferrari et Espejo y (B) H. zamudioi Espejo, López-Ferrari et I. Ramírez. Fotografías A. Espejo. 

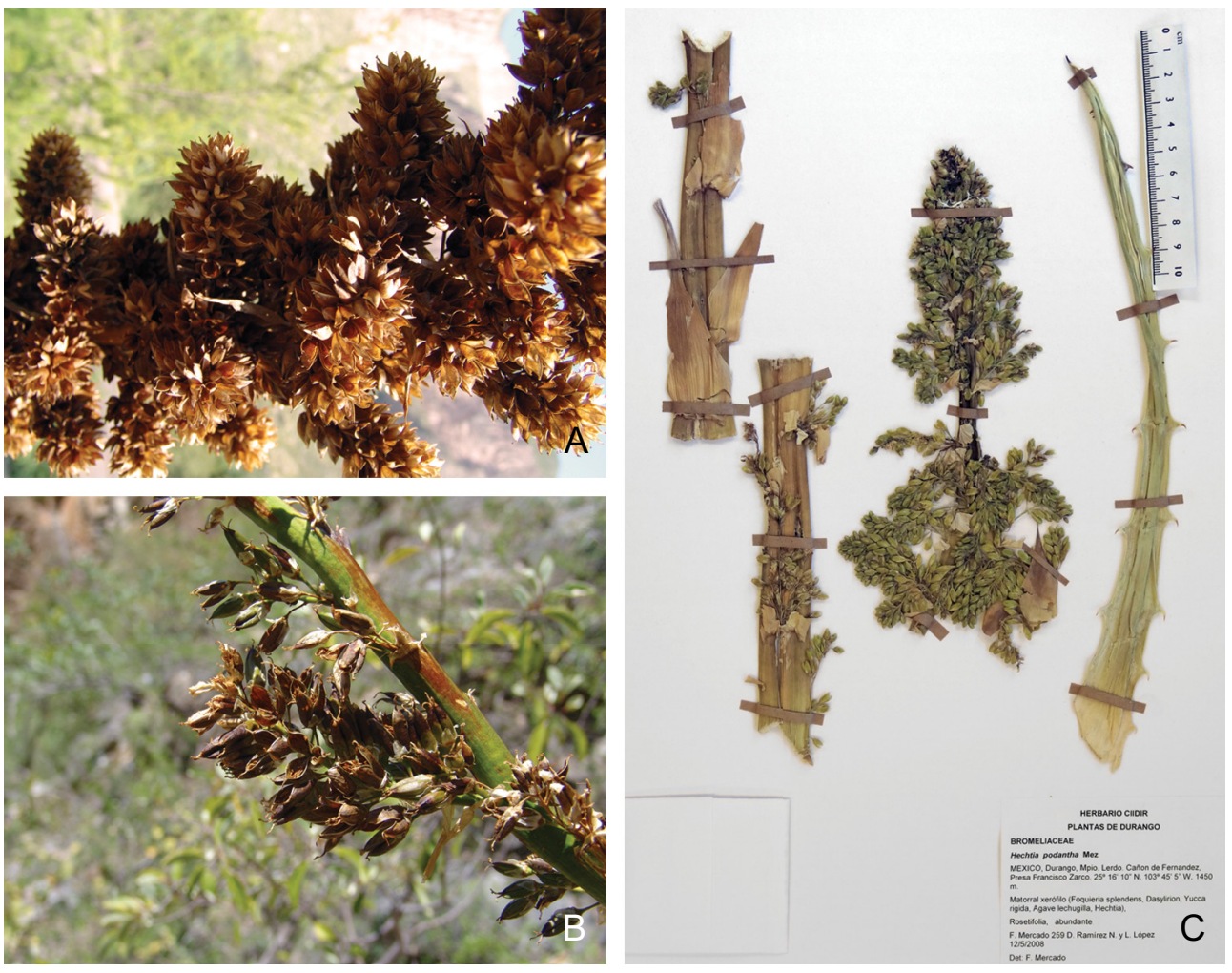

Fig. 4. Infrutescencias de (A) Hechtia mapimiana López-Ferrari et Espejo y (B) H. zamudioi Espejo, López-Ferrari et I. Ramírez. (C) Holotipo de H. mapimiana López-Ferrari et Espejo. Fotografías A. Espejo.

limestone, 7.1 miles $\mathrm{W}$ of hwy 40, on road to presa Francisco Zarco along río Nazas, $25^{\circ} 16^{\prime} \mathrm{N}, 103^{\circ} 45^{\prime} \mathrm{W}, 4300 \mathrm{ft}$, with Aloysia, Leucaena, Yucca thompsoniana, Agave spp., Larrea, etc., 15.VIII.1973, planta femenina, J. Henrickson 12441 (MEXU, MO, US).

Distribución y hábitat. Hechtia mapimiana es endémica de la sierra de Mapimí en el parque estatal Cañón de Fernández, del municipio de Lerdo, en el estado de Durango. La nueva especie crece en grupos más o menos compactos, sobre peñas y riscos con vegetación de matorral xerófilo, entre 1400 y 1750 m s.n.m. Florece y fructifica entre los meses de mayo y julio. Dado que la especie crece abundantemente en los acantilados del cañón del río Nazas y sus afluentes, en sitios poco accesibles y dentro de un área natural protegida, pensamos que no enfrenta problemas de conservación. 
Etimología. El nombre de la especie alude a la región geográfica donde la misma habita y de la cual es endémica.

Hechtia mapimiana presenta algunas semejanzas, tanto vegetativas como florales, con H. zamudioi Espejo, López-Ferrari \& I. Ramírez, taxon endémico de la cuenca del río Estórax, en el estado de Querétaro (Espejo Serna et al., 2008). Ambas especies habitan formando colonias en matorrales xerófilos que prosperan sobre escarpadas paredes de rocas calizas (Fig. 2) en intervalos altitudinales similares y en sendos casos las plantas presentan rosetas de color glauco a azuloso y tienen flores verdes. Sin embargo, existen entre ellas claras diferencias, como puede apreciarse en el Cuadro 1 y en las figuras 3 y 4.

Por otra parte, cabe comentar aquí que para el estado de Durango tenemos registradas además de la especie aquí propuesta a Hechtia texensis S. Watson y $H$. subalata L. B. Sm., las cuales difieren de H. mapimiana por presentar las flores blancas y las plantas y hojas más pequeñas.

Cuadro 1. Características comparativas de Hechtia mapimiana Espejo \& López-Ferrari y $H$. zamudioi Espejo, López-Ferrari \& I. Ramírez.

\begin{tabular}{|c|c|c|}
\hline & Hechtia mapimiana & Hechtia zamudioi \\
\hline Hojas & $\begin{array}{l}\text { cilíndrico-cónicas hacia el } \\
\text { ápice }\end{array}$ & aplanadas hacia el ápice \\
\hline Vainas foliares & $\begin{array}{l}\text { pardas claras en el haz, pardas } \\
\text { oscuras en el envés }\end{array}$ & $\begin{array}{l}\text { amarillas pajizas en el haz y } \\
\text { en el envés }\end{array}$ \\
\hline Inflorescencia pistilada & dos veces dividida & una vez dividida \\
\hline Sépalos flor pistilada & $\begin{array}{l}2.5 \mathrm{~mm} \text { de ancho, blancos con } \\
\text { tintes purpúreos }\end{array}$ & $1.2-1.4 \mathrm{~mm}$ de ancho, verdes \\
\hline Estaminodios & anteras ausentes & con anteras vestigiales \\
\hline Inflorescencia estaminada & $\begin{array}{l}\text { dos a tres veces dividida, } \\
\text { ramas fasciculadas }\end{array}$ & $\begin{array}{l}\text { una a dos veces dividida, } \\
\text { ramas no fasciculadas }\end{array}$ \\
\hline Sépalos flor estaminada & $\begin{array}{l}1.5-1.7 \mathrm{~mm} \text { de ancho, blancos } \\
\text { con tintes rosados a purpúreos }\end{array}$ & $\begin{array}{l}\text { 2-3.5 } \mathrm{mm} \text { de ancho, verdes, } \\
\text { hialinos }\end{array}$ \\
\hline Filamentos & $3.2-3.5 \mathrm{~mm}$ de largo & 6-7 mm de largo \\
\hline Anteras & 4.2-4.5 mm de largo, verdes & ca. $3 \mathrm{~mm}$ de largo, amarillas \\
\hline Distribución & Durango & Querétaro \\
\hline $\begin{array}{l}\text { Provincias biogeográficas } \\
\text { (Anónimo, 1997) }\end{array}$ & $\begin{array}{l}\text { Altiplano Norte } \\
\text { (Chihuahuense) }\end{array}$ & $\begin{array}{l}\text { Altiplano Sur (Zacatecano- } \\
\text { Potosino) }\end{array}$ \\
\hline
\end{tabular}




\section{AGRADECIMIENTOS}

Deseamos expresar nuestro agradecimiento a Socorro González Elizondo, por habernos facilitado material del sexo masculino de la nueva especie y a Lorena López Enríquez, encargada del herbario CIIDIR, por el préstamo del ejemplar designado como holotipo de la nueva especie. A los curadores de los herbarios IEB, MEXU, MO, UAMIZ y US por las facilidades para consultar sus colecciones.

\section{LITERATURA CITADA}

Anónimo, 1997. Provincias biogeográficas de México. Escala 1:4 000 000. Comisión Nacional para el Conocimiento y Uso de la Biodiversidad, México, D.F., México.

Espejo-Serna, A., A. R. López-Ferrari e I. Ramírez-Morillo. 2008. Dos nuevas especies de Hechtia (Bromeliaceae; Pitcairnioideae) del centro de México. Acta Bot. Mex. 83: 49-61. 